\title{
Dynamics of the order parameter in a photoexcited Peierls chain
}

\author{
Yong Wang, ${ }^{1}$ Wei-Qiang Chen, ${ }^{2}$ and Fu-Chun Zhang ${ }^{3,1}$ \\ ${ }^{1}$ Department of Physics, The University of Hong Kong, Hong Kong SAR, China \\ ${ }^{2}$ Department of Physics, South University of Science and Technology of China, China \\ ${ }^{3}$ Department of Physics, Zhejiang University, China
}

(Received 23 April 2014; revised manuscript received 2 September 2014; published 6 November 2014)

\begin{abstract}
The photoexcited dynamics of the order parameter in a Peierls chain is investigated by using a microscopic quantum theory in the limit where the hot electrons may establish themselves into a quasi-equilibrium state described by an effective temperature. The optical phonon mode responsible for the Peierls instability is coupled to the electron subsystem, and its dynamic equation is derived in terms of the density matrix technique. Recovery dynamics of the order parameter is obtained, which reveals a number of interesting features including the change of oscillation frequency and amplitude at the phase-transition temperature and the photoinduced switching of the order parameter.
\end{abstract}

DOI: 10.1103/PhysRevB.90.205110

PACS number(s): 71.45.Lr, 05.70.Ln, 78.47.da

\section{INTRODUCTION}

The concept of order parameter, which characterizes various phases in condensed matter, plays an essential role in modern phase-transition theory [1]. One of the recent focuses is the dynamics of the order parameter in nonequilibrium processes, which is of interest in both basic and applied physics. Micromagnetics for magneto-electronics devices is an excellent example of such applications [2]. The nonequilibrium dynamics of the charge density wave (CDW) order has recently been extensively investigated by using optical pump-probe spectroscopy [3-6] and time-dependent angleresolved photoemission spectroscopy (trARPES) [7-12]. In these experiments, the samples are initially excited by a pump laser pulse, and the electronic dynamics is then observed either by probing the laser pulse or by using the ARPES technique. Depending upon the pump fluence, there are two types of electronic dynamics. In the low-pump-fluence case, the CDW order is only slightly perturbed and the collective modes in the CDW phase are excited. If the pump fluence is high enough to destroy (meltdown) the CDW order, a dynamic phase transition occurs and the recovery dynamics of the initial CDW order will be observed. These two types of dynamics are not mutually exclusive but closely related, since the recovery of the CDW order is always accompanied with the excitations of the collective mode [3-12]. The study of these nonequilibrium dynamics processes may provide unique insight to identify the formation mechanism of the CDW order [7,10,11].

In comparison with the experimental progress, the theoretical efforts to understand and describe these phenomena are still very limited. One approach is the time-dependent Ginzburg-Landau (TDGL) equation [6]. As a phenomenological description, although it is effective in describing some experiments, it does not give the microscopic details of the dynamics. Considering the complex microscopic interactions in these CDW materials, it is helpful and desirable to develop a microscopic theory for a more fundamental and complete understanding of the dynamic processes. Such a necessity has been exemplified in the study of the optical conductivity of the Hubbard-Holstein model [13].

In this paper, we consider the photoexcited dynamics of the Peierls-type CDW systems based on a microscopic model.
We investigate the half-filled Peierls chain and show how the nonequilibrium dynamics of the order parameter emerges from the microscopic interaction and how to obtain the coefficients in the TDGL equation from the basic parameters in the microscopic model. This prototype study provides a microscopic picture to understand the nonequilibrium quantum dynamics in CDW systems.

\section{THEORETICAL MODEL}

\section{A. System Hamiltonian}

We consider a Peierls chain consisting of $2 N$ monovalent atoms with the periodic boundary condition as depicted in Fig. 1. The equilibrium positions of the atoms at high temperature are shown in configuration (I). The deviation of the $i$ th atom from its equilibrium position is denoted as $u_{i}$. The system Hamiltonian, or the Su-Schrieffer-Heeger model, is defined as [14]

$$
\begin{aligned}
H= & -\sum_{i, \sigma}\left(t_{i, i+1} c_{i+1, \sigma}^{\dagger} c_{i, \sigma}+\text { H.c. }\right) \\
& +\sum_{i} \frac{1}{2} M \dot{u}_{i}^{2}+\sum_{i} \frac{1}{2} K\left(u_{i+1}-u_{i}\right)^{2},
\end{aligned}
$$

where we only consider the nearest-neighbor hopping of the electrons. $c_{i, \sigma}^{\dagger}\left(c_{i, \sigma}\right)$ is the creation (annihilation) operator for the electron at the $i$ th atom with spin $\sigma, M$ is the atom mass, and $K$ characterizes the force constant to pull the atom back to its equilibrium position. The hopping integral $t_{i, i+1}$ is a function of $\delta u_{i}=u_{i}-u_{i+1}$. Since $\delta u_{i}$ is usually small, we can expand $t_{i, i+1}$ to first order in $\delta u_{i}$, i.e., $t_{i+1, i}=t_{0}-\alpha\left(u_{i+1}-u_{i}\right)$, where $t_{0}$ is the hopping integral in the absence of lattice distortion.

At low temperatures, the half-filled chain will be dimerized, as shown in configuration (II), due to the Peierls instability. The unit cell is enlarged to include two atoms in this phase, so the lattice constant becomes $2 a$ with $a$ being the nearest-neighboratom distance in the undimerized state. In the following, we use the enlarged unit cell for convenience, where the unit cell is denoted with $m=1,2, \ldots, N$, and the two atoms in one unit cell are denoted $A$ and $B$, respectively. Then we move 


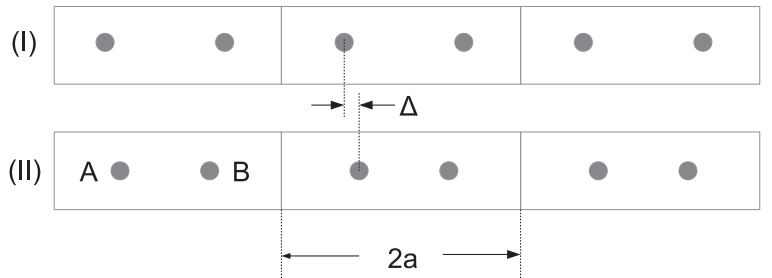

FIG. 1. (I) Undistorted and (II) distorted configurations of a Peierls chain. The chain consists of $N$ unit cells with two atoms $(\eta=A, B)$ in each unit cell. The lattice constant is $2 a$, and the displacement of the distorted $A$-type atoms is the order parameter $\Delta$.

to the reciprocal in the reduced Brillouin zone space, and the Hamiltonian reads (Appendix A)

$$
\begin{gathered}
H_{p}=\frac{1}{2} \sum_{\mu, q}\left(\widehat{P}_{\mu, q}^{*} \widehat{P}_{\mu, q}+\omega_{\mu, q}^{2} \widehat{Q}_{\mu, q}^{*} \widehat{Q}_{\mu, q}\right), \\
H_{e}=-t_{0} \sum_{k, \sigma}\left[\left(1+e^{-2 i k a}\right) c_{A, k, \sigma}^{\dagger} c_{B, k, \sigma}+\text { H.c. }\right], \\
H_{\mathrm{ep}}=\sum_{\mu, q, k, \sigma}\left(g_{\mu, q, k} \widehat{Q}_{\mu, q} c_{A, k, \sigma}^{\dagger} c_{B, k-q, \sigma}+\text { H.c. }\right),
\end{gathered}
$$

where $H_{p}$ and $H_{e}$ are the Hamiltonian of the phonon and electron subsystems, respectively, $H_{\mathrm{ep}}$ describes the electronphonon interaction (EPI), and $q, k \in\left[-\frac{\pi}{2 a}, \frac{\pi}{2 a}\right]$ are the wave vectors in the reduced zone. $\mu=\mathrm{a}$, o denotes the acoustic and optical phonons, respectively. $\widehat{Q}_{\mu, q}$ and $\widehat{P}_{\mu, q}$ are the canonical coordinates and momentum of the phonons, respectively, and they satisfy the quantization condition $\left[\widehat{Q}_{\mu, q}, \widehat{P}_{\mu^{\prime}, q^{\prime}}\right]=$ $i \hbar \delta_{q,-q^{\prime}} \delta_{\mu, \mu^{\prime}} . \omega_{\mu, q}$ is the phonon energy. $c_{\eta, k, \sigma}^{\dagger}\left(c_{\eta, k, \sigma}\right)$ is the Fourier transformation of $c_{\eta, m, \sigma}^{\dagger}\left(c_{\eta, m, \sigma}\right)$, with $\eta=A, B \cdot g_{\mu, q, k}$ represents the spin-independent electron-phonon interaction.

For the half-filled Peierls chain, the nesting wave vector is $\mathbf{K}_{\text {nest }}=0$ in the reduced zone. So the phonon mode which condenses in the Peierls phase transition is the optical one with $q=0$, denoted as $(\mathrm{o}, 0)$. In the pump-probe technique, the electron subsystem is excited to a highly nonequilibrium state by the strong pump laser. Then it reaches quasi-equilibrium state through electron-electron scattering on a femtosecond timescale [8], which could be characterized by an effective temperature $T_{e}$. On the other hand, the lattice is nearly unaffected in the thermalization process because of the much larger mass of the ions. It is reasonable to assume that the lattice keeps its original temperature $T_{l}$. In the Peierls phase, the distortion of the lattice is due to the electron-phonon coupling. After the electrons are heated by the pumping laser, the ions are not at the configuration with lowest energy any more, and they will start to move towards the configuration with lower energy. Then the eigenstates of the electrons will vary due to the movement of the ions, and the hot electrons will transfer the extra energy to the lattice, which could be considered as a heat bath with temperature $T_{l}$, until its temperature $T_{e}$ reduces to $T_{l}$.

Based on this picture, two equations will be used to describe the dynamics of the photoexcited Peierls chain. Since we are more interested in the dynamics of the lattice, we will first derive the equation by describing the dynamics of the condensate of phonon mode $(\mathrm{o}, 0)$, with its coupling to the electrons in the microscopic Hamiltonian through the density matrix technique. Then we introduce a phenomenological equation to describe the temperature decay of the electron subsystem.

\section{B. Dynamical equations for condensed phonon}

In the following, we denote the operators $\left(\widehat{Q}_{o, 0}, \widehat{P}_{o, 0}\right)$ for the phonon mode $(\mathrm{o}, 0)$ as $(\widehat{\mathrm{Q}}, \widehat{P})$, and the corresponding frequency as $\Omega^{2} \equiv \omega_{o, 0}^{2}=\frac{4 K}{M}$. With the density matrix $\rho$ of the phonon mode $(0,0)$, the expectation value of the operators are $\mathcal{Q}(t)=\operatorname{Tr}[\widehat{\mathrm{Q}} \rho]$, and $\mathcal{P}(t)=\operatorname{Tr}[\widehat{\mathrm{P}} \rho]$. In order to investigate the dynamics of $\mathcal{Q}$, we study how it evolves from time $t^{\prime}$ to $t=t^{\prime}+\delta t$ within a small $\delta t$. For simplicity, we denote $\mathcal{Q}_{0} \equiv \mathcal{Q}\left(t^{\prime}\right)$ and define a new canonical coordinate $\widehat{\mathrm{q}}$ as $\widehat{\mathrm{q}} \equiv \widehat{\mathrm{Q}}-\mathcal{Q}_{0}$. Then the Hamiltonian of the phonon mode $(\mathrm{o}, 0)$ becomes

$$
H_{S}=\frac{1}{2}\left[\widehat{\mathrm{P}}^{2}+\Omega^{2}\left(\widehat{\mathrm{q}}^{2}+2 \mathcal{Q}_{0} \widehat{\mathrm{q}}+\mathcal{Q}_{0}^{2}\right)\right] .
$$

The coupling term for the electrons and the phonon mode $(0,0)$ contained in Eq. (4) is also decomposed into two terms $-\mathcal{Q}_{0} \widehat{F}$ and $-\widehat{q} \otimes \widehat{F}$, where

$$
\widehat{\mathrm{F}}=\sqrt{\frac{2 \alpha^{2}}{N M}} \sum_{k, \sigma}\left[\left(1-e^{-2 i k a}\right) c_{A, k, \sigma}^{\dagger} c_{B, k, \sigma}+\text { H.c. }\right] .
$$

In the small time interval $\left[t^{\prime}, t\right]$, the effect of the Peierls distortion on the hot electrons is dominated by the term $-\mathcal{Q}_{0} \widehat{F}$, which gives the electron Hamiltonian as $H_{B}=H_{e}-\mathcal{Q}_{0} \widehat{\mathrm{F}}$. On the other hand, the term $V \equiv-\widehat{\mathrm{q}} \otimes \widehat{\mathrm{F}}$ gives the quantum dynamics of the phonon mode $(0,0)$ driven by the hot electrons.

We now focus on the quantum dynamics of the phonon mode $(0,0)$ during $\left[t^{\prime}, t\right]$. In the two-temperature scenario, we assume that the quasi-equilibrium state of the hot electrons has been established with the effective temperature $T_{e}$. Then the phonon dynamics can be calculated with the standard "system plus reservoir" paradigm, where the system Hamiltonian is $H_{S}$, the electron reservoir Hamiltonian is $H_{B}$, and the interaction is $V$. To second order of the interaction $V$, the equation of motion of the density matrix of the phonon mode $(0,0)$ in the interaction picture is [15] (Appendix B)

$$
\begin{aligned}
\frac{d \widetilde{\rho}(t)}{d t}= & -\frac{1}{i \hbar}\langle\widetilde{\mathrm{F}}\rangle_{B}\left[\widetilde{\mathbf{q}}(t), \widetilde{\rho}\left(t^{\prime}\right)\right] \\
& -\frac{1}{\hbar^{2}} \int_{t^{\prime}}^{t} d \tau \mathcal{J}(t-\tau)[\widetilde{\mathrm{q}}(t),[\widetilde{\mathbf{q}}(\tau), \widetilde{\rho}(\tau)]] \\
& -\frac{i}{\hbar^{2}} \int_{t^{\prime}}^{t} d \tau \mathcal{K}(t-\tau)[\widetilde{\mathbf{q}}(t),\{\widetilde{\mathbf{q}}(\tau), \widetilde{\rho}(\tau)\}] .
\end{aligned}
$$

Here, $\approx$ denotes the operators in the interaction picture, and $\langle\ldots\rangle_{B} \equiv \operatorname{Tr}_{B}\left[\ldots \tilde{\rho}_{B}\right]$ denotes the expectation value over the reservoir density matrix $\tilde{\rho}_{B} \cdot \mathcal{J}(t-\tau)$ and $\mathcal{K}(t-\tau)$ are the real and imaginary part of the correlation function $\langle\widetilde{\mathrm{F}}(t) \widetilde{\mathrm{F}}(\tau)\rangle_{B}$ respectively. Based on physical considerations, the above equation holds if $\delta t$ is larger than the correlation time of the kernels $\mathcal{J}(t-\tau)$ and $\mathcal{K}(t-\tau)$ but still small enough in order to assume that $\mathcal{Q}$ and $T_{e}$ are unchanged during the time interval. This can be justified since the lattice dynamics is on 
the picosecond timescale while the kernel correlation time is on the order of femtoseconds.

With Eq. (7) for the density matrix, the equations for $\dot{\mathcal{Q}}$ and $\dot{\mathcal{P}}$ during the small time interval $\left[t^{\prime}, t\right]$ are (Appendix C)

$$
\begin{gathered}
\dot{\mathcal{Q}}(t)=\mathcal{P}(t) \\
\dot{\mathcal{P}}(t)=-\Omega^{2} \mathcal{Q}(t)+\mathcal{F}(t)-\frac{2}{\hbar} \int_{t^{\prime}}^{t} d \tau \mathcal{K}(t-\tau) \mathrm{q}(\tau) .
\end{gathered}
$$

Here, $\mathcal{F}=\langle\widetilde{\mathrm{F}}\rangle_{B}$. The displacement of the ions in real space is $\Delta \equiv u_{A, m}=-u_{B, m}=\frac{\mathcal{Q}}{\sqrt{2 N M}}$. By applying the partial integration to the convolution integral in Eq. (9) and dropping the term proportional to $\mathrm{q}(t)$ which vanishes for $\delta t \rightarrow 0$, we get an equation for the order parameter $\Delta$ as

$$
\ddot{\Delta}+\int_{t^{\prime}}^{t} d \tau \gamma(t-\tau) \dot{\Delta}(\tau)-\frac{\mathrm{f}}{M}=0 .
$$

Here, the damping kernel $\gamma(t)$ is related to the kernel $\mathcal{K}(t)$ via their Fourier transformations $\gamma(\omega)=-\frac{2}{\hbar} \frac{\mathcal{K}(\omega)}{\omega}$, and $\mathrm{f}$ is the force conjugated to the order parameter. Equation (10) takes the similar form as the TDGL equation, but its validity is not restricted to the temperature range near the phase transition. Furthermore, the damping kernel $\gamma$ and the force $f$ are not given phenomenologically but are determined from the parameters in the Peierls model including $K, M, t_{0}, \alpha$, and $T_{e}$. This enables us to understand microscopic origin of the order-parameter dynamics directly.

In our treatment here, only the quantum dynamics of the phonon mode $(0,0)$ which is responsible for the Peierls stability has been investigated, and the other phonon modes have been simply treated as a thermal bath to cool down the hot electrons. This implies that the dynamics of other modes such as small- $q$ modes has been neglected and possible polaronic effects are not considered. A uniform order parameter has been assumed for the dynamics after the photoexcitation, and possible excitations of solitons and polarons [16-18] have been excluded in our study here. This simplification is ideal in comparison with the more complicated realistic cases but is helpful to capture the key physics picture that we are more interested in.

\section{Force and damping coefficient}

The force $\mathrm{f}$ in Eq. (10) consists of two terms (Appendix E),

$$
\mathrm{f}=-4 K \Delta+\frac{1}{N} \sum_{k} \frac{16 \alpha^{2} \Delta \sin ^{2} k a}{\Lambda_{k}} \tanh \frac{\beta \Lambda_{k}}{2},
$$

where $\beta=1 /\left(k_{B} T_{e}\right)$. The first term is the elastic force between the ions, and the second term comes from the coupling to the electron subsystem. The spin index has been taken into account by a factor 2 in the summation. The dispersions of the electron bands are $\epsilon_{ \pm, k, \sigma}= \pm \Lambda_{k}$ with $\Lambda_{k}=\left(4 t_{0}^{2} \cos ^{2} k a+16 \alpha^{2} \Delta^{2} \sin ^{2} k a\right)^{1 / 2}$. It is easy to check that the force $\mathrm{f}$ is related to the free energy $\mathcal{E}$ as $\mathrm{f}=-\partial \mathcal{E} / \partial \Delta$, with

$$
\mathcal{E}\left(\Delta, T_{e}\right)=2 K \Delta^{2}-\frac{1}{N \beta} \sum_{k} \ln \left(2+e^{-\beta \Lambda_{k}}+e^{\beta \Lambda_{k}}\right) .
$$

The Fourier transformation of the kernel functions $\mathcal{J}(\omega), \mathcal{K}(\omega), \gamma(\omega)$ can be calculated based on the microscopic model. Especially, we have (Appendix E)

$$
\begin{aligned}
\gamma(\omega)= & \frac{2}{\hbar \omega} \sum_{k}\left|h_{k}\right|^{2} \tanh \frac{\beta \Lambda_{k}}{2}\left[\delta\left(\omega-\frac{2 \Lambda_{k}}{\hbar}\right)\right. \\
& \left.-\delta\left(\omega+\frac{2 \Lambda_{k}}{\hbar}\right)\right],
\end{aligned}
$$

which is is an even function of $\omega$. Here, the coefficients $\left|h_{k}\right|^{2}$ are (Appendix E)

$$
\left|h_{k}\right|^{2}=\frac{8 \alpha^{2}}{N M} \frac{t_{0}^{2} \sin ^{2} k a \cos ^{2} k a}{t_{0}^{2} \cos ^{2} k a+4 \alpha^{2} \Delta^{2} \sin ^{2} k a} .
$$

Then the damping kernel in Eq. (10) becomes

$$
\gamma(t)=\int_{-\infty}^{+\infty} d \omega \gamma(\omega) \cos (\omega t)
$$

In a typical Peierls system, $t_{0}$ is on the order of eV, while $\alpha \Delta$ and $1 / \beta$ are on the order of meV. We see that $\gamma(\omega)$ depends on the order parameter $\Delta$ and the temperature $T_{e}$. The maximum of $\Lambda_{k}$ is $2 t_{0}$ since $t_{0} \gg \alpha \Delta$, then the cutoff frequency of $\gamma(\omega)$ will be $\omega_{c}=4 t_{0} / \hbar$. Since $\gamma(\omega)$ is affected merely in the low-frequency range characterized by the energy $1 / \beta$ and $\alpha \Delta$ which is much smaller than the cutoff energy $\hbar \omega_{c}, \gamma(t)$ may be approximated to be independent on $T_{e}$ and $\Delta$ in the following calculations without bringing qualitative error. The correlation time of the damping kernel $\gamma(t)$ is estimated as $t_{c}=\frac{2 \pi}{\omega_{c}}=\frac{\pi \hbar}{2 t_{0}}$, therefore $t_{c}$ is on the order of femtoseconds, which is much shorter than the picosecond timescale of the order-parameter dynamics. This suggests that the Markov approximation can be applied to the damping term in Eq. (10) by replacing $\dot{\Delta}(\tau)$ with $\dot{\Delta}(t)$. Then Eq. (10) is reduced to

$$
\ddot{\Delta}+\Gamma \dot{\Delta}-\frac{f}{M}=0,
$$

where $\Gamma=\int_{t^{\prime}}^{t} d \tau \gamma(t-\tau)=\int_{0}^{\delta t} d \tau \gamma(\tau)$ with $\delta t>t_{c}$.

In order to get a qualitative estimation of $\Gamma$, we consider the case $\Delta=0$ and $\beta \Lambda_{k} \gg 1$, which gives $\left|h_{k}\right|^{2}=\frac{8 \alpha^{2}}{N M} \sin ^{2} k a$ and $\Lambda_{k}=2 t_{0} \cos k a \cdot \gamma(\omega)$ is calculated as

$$
\gamma(\omega)=\frac{4 \alpha^{2}}{\pi M t_{0}} \frac{\sqrt{1-\left(\omega / \omega_{c}\right)^{2}}}{|\omega|}
$$

for $|\omega| \leqslant \omega_{c}$. Then one gets $\gamma(\omega) \sim \frac{4 \alpha^{2}}{\pi M t_{0} \omega_{c}} \sim \frac{\hbar \alpha^{2}}{\pi M t_{0}^{2}}, \gamma(t) \sim$ $\gamma(\omega) \omega_{c} \sim \frac{4 \alpha^{2}}{\pi M t_{0}}$, and $\Gamma \sim \gamma(t) t_{c} \sim \frac{\hbar \alpha^{2}}{M t_{0}^{2}}$. So we can estimate the damping coefficient $\Gamma$ in Eq. (15) from the basic parameters $M, t_{0}, \alpha$ except for a proportional factor.

\section{Two-temperature scenario}

In our simplified treatment, all the phonon modes except $(0,0)$ form a thermal reservoir with temperature $T_{l}$. The electron subsystem will dissipate extra energy into the reservoir through electron-phonon coupling on the picosecond timescale. Although this dissipative process can also been described by the quantum dynamics method based on the microscopic Hamiltonian, a more efficient way is to exploit 
the two-temperature scenario considering that the thermalization process of hot electrons happens on the femtosecond timescale. This thermalization approximation is usually reasonable enough to describe the electron relaxation process on the subpicosecond timescale [8] and has been exploited to understand the trAPRES results on cuprates qualitatively [19]. For simplicity, we assume that the electronic temperature $T_{e}$ decays towards $T_{l}$ in the exponential form, i.e..

$$
T_{e}(t)=T_{l}+\left(T_{e}(0)-T_{l}\right) e^{-t / \kappa},
$$

where the initial electronic temperature $T_{e}(0)$ depends on the fluence of the pump laser, and the decay time $\kappa$ is a fitting parameter. Equations (15) and (16) are the two basic equations in our theory to describe the subpicosecond dynamics of the photoexcited Peierls chain.

\section{CALCULATION RESULTS}

We perform some calculations for demonstration based on the theory established above. We choose the following parameters, $t_{0}=2 \mathrm{eV}, \alpha=0.167 \mathrm{eV} / \AA, M=1.35 \times 10^{-26} \mathrm{~kg}$, and $K=1.2 \mathrm{~kg} / \mathrm{s}^{2}$, which gives the oscillation frequency $\Omega=6 \pi \mathrm{THz}$. In the numerical calculation, we consider a system with $N=2000$ unit cells. The free energy $\mathcal{E}\left(\Delta, T_{e}\right)$ in Eq. (12) calculated for $\Delta \in[-0.3,0.3] \AA$ and $T_{e} \in[1,800] \mathrm{K}$ are shown in Fig. 2(a). At high temperature, the minimum of $\mathcal{E}$ is at $\Delta=0$ corresponding to the undistorted configuration (I); while at low temperature, $\mathcal{E}$ has the form of doublewell potential, and the distorted configuration (II) is stable. Figure 2(b) shows the $T_{e}$ dependence of the stable order
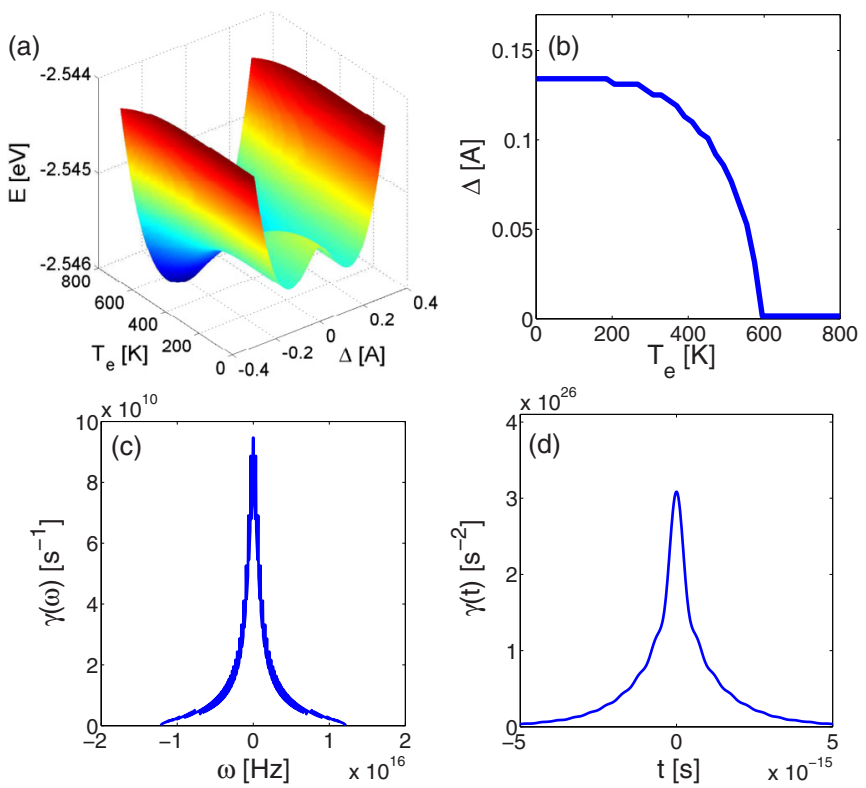

FIG. 2. (Color online) (a) The free energy per atom $\mathcal{E}$ as a function of electronic temperature $T_{e}$ and order parameter $\Delta$. (b) The order parameter $\Delta$ as a function of electronic temperature $T_{e}$, obtained by minimizing the free energy density $\mathcal{E}$. [(c), (d)] The damping kernel $\gamma(t)$ and its Fourier transformation $\gamma(\omega)$ calculated for $\Delta=0$ and $T_{e}=1000 \mathrm{~K}$. The basic model parameters for calculations here are given as $t_{0}=2 \mathrm{eV}, \alpha=0.167 \mathrm{eV} / \AA, M=1.35 \times 10^{-26} \mathrm{~kg}$, $K=1.2 \mathrm{~kg} / \mathrm{s}^{2}$, and $N=2000$. parameter $\Delta(>0)$, which is in fact the solution of the equation

$$
4 K=\frac{1}{N} \sum_{k} \frac{16 \alpha^{2} \sin ^{2} k a}{\Lambda_{k}} \tanh \frac{\beta \Lambda_{k}}{2},
$$

obtained by setting $f=0$ in Eq. (11). The phase-transition temperature is found to be about $600 \mathrm{~K}$ for the chosen parameters here. The solutions thus recover the mean-field results for the CDW phase transition [20].

With the given parameters, the damping kernel function $\gamma(t)$ and its Fourier transformation $\gamma(\omega)$ are also calculated with their expressions for $\Delta=0$ and $T_{e}=1000 \mathrm{~K}$, as shown in Figs. 2(c) and 2(d). According to the qualitative estimation above, the cutoff frequency $\omega_{c}=1.2 \times 10^{16} \mathrm{~Hz}$, and $\gamma(\omega) \sim$ $1.7 \times 10^{9} \mathrm{~s}^{-1}$; while the correlation time $t_{c}=5.2 \times 10^{-16} \mathrm{~s}$, and $\gamma(t) \sim 2 \times 10^{25} \mathrm{~s}^{-2}$. This indeed captures the qualitative features of the calculated damping kernel. Furthermore, the damping coefficient in Eq. (15) is calculated as $\Gamma=0.25 \mathrm{ps}^{-1}$ by integrating $\gamma(t)$ from 0 to 5 fs. This parameter will be exploited in the following dynamics simulations.

Now we simulate the dynamics of the order parameter $\Delta$ based on Eqs. (15) and (16). The decay time of $T_{e}$ is chosen to be $\kappa=5$ ps. In the calculation, we discretize the equations with $\delta t=5$ fs between two time steps. The initial order parameter $\Delta(0)$ is determined from Eq. (17) with given lattice temperature $T_{l}$, and $\dot{\Delta}(0)=0$. The initial electronic temperature $T_{e}(0)$ depends on the fluence of the pump laser and is an input parameter in our simulations. The simulations with various $T_{l}$ and $T_{e}(0)$ are performed. The results with three different $T_{l}=100,300,500 \mathrm{~K}$ and fixed $T_{e}(0)=1200 \mathrm{~K}$ are depicted in Fig. 3(a), and the results with three different $T_{e}(0)=800,1000,1200 \mathrm{~K}$ and fixed $T_{l}=200 \mathrm{~K}$ are shown in Fig. 3(b). Several features of the dynamics can be observed in the figures. First, the oscillation frequency of the order parameter is lower than the bare frequency $\Omega=6 \pi \mathrm{THz}$ of the phonon mode $(0,0)$, because of the coupling between the phonons and electrons. Since the damping effect is of second order in EPI in our theory, it is smaller than the restoring force which is of first order in EPI. This gives the damping oscillation in our calculations which agrees with the
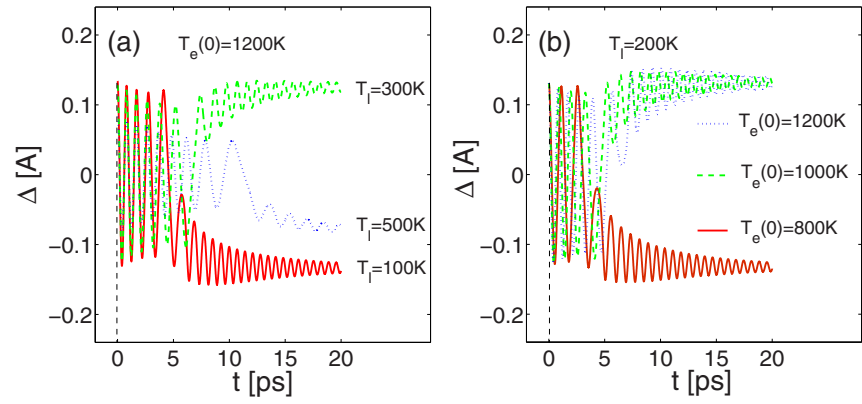

FIG. 3. (Color online) (a) Order-parameter dynamics for three different lattice temperatures $T_{l}=100,300,500 \mathrm{~K}$ with fixed initial electronic temperature $T_{e}(0)=1200 \mathrm{~K}$. (b) Order-parameter dynamics for three different initial electronic temperatures $T_{e}(0)=$ $800,1000,1200 \mathrm{~K}$ with fixed lattice temperature $T_{l}=200 \mathrm{~K}$. The basic model parameters are the same as in Fig. 2, and the damping coefficient $\Gamma=0.25 \mathrm{ps}^{-1}$, the electronic temperature decay time $\kappa=5 \mathrm{ps}$, and the discretized time step $\delta t=5 \mathrm{fs}$. 
experimental observations [3-12]. Second, the dynamics can be divided into two regions. When the electronic temperature $T_{e}$ is above the phase-transition temperature, the amplitude of the oscillation is large while frequency is low; when $T_{e}$ is below the phase-transition temperature, the order parameter is trapped in one of the double wells, and it oscillates with smaller amplitude but higher frequency. This reflects the symmetry breaking when the electronic temperature crosses the phase-transition temperature. Third, switching of the order parameter is possible in some cases as long as $T_{e}(0)$ is larger than the phase-transition temperature. This suggests the possibility to control the Peierls systems optically on the picosecond timescale for practical applications.

\section{CONCLUSION}

The photoexcited dynamics of the order parameter in a Peierls chain has been investigated within the microscopic quantum theory in the limit that the electron subsystem can be treated as a quasi-equilibrium state with an exponentially decaying effective temperature. The optical phonon mode responsible for the Peierls distortion is coupled to the electron subsystem, and its dynamical equation is established with the help of the density matrix technique. The driven force acting on the order parameter and the damping coefficient are obtained in terms of the microscopic parameters in the Peierls model. We further apply the theory to simulate the recovery dynamics of the order parameter in the photoexcited Peierls chain. It is found that the oscillation frequency and amplitude will be significantly changed when the effective electronic temperature crosses the phase-transition temperature, and switching of the order parameter may happen. Although the order parameter has been assumed to be uniform where the possible excitations of solitons and polarons are not considered here, our theory is able to give the microscopic physics picture to describe the recovery dynamics and collective oscillations of the CDW order parameter, which is more relevant to quasi-onedimensional Peierls-type CDW materials and incommensurate one-dimensional Peierls chains where soliton excitations are absent. Our theory is also not restricted to the temperature range near the phase transition as in the phenomenological Ginzburg-Landau theory. The approximations in our semiphenomenological approach can be benchmarked or improved by more sophisticated approaches such as dynamical mean-field theory [21] and Keldysh perturbation theory [22]. Thus our theory serves as the bridge connecting the phenomenological GL approach and the full microscopic approaches and can be further generalized to understand the photoexcited dynamics in more complicated Peierls-type systems and might have potential applications.

\section{ACKNOWLEDGMENTS}

This work was supported in part by the Hong Kong's University Grant Council via Grant No. AoE/P-04/08. W.Q. Chen was partly supported by NSFC Project No. 11204186, and F.C. Zhang was partly supported by NSFC Project No. 11274269.

\section{APPENDIX A: MODEL HAMILTONIAN}

\section{Lattice Hamiltonian}

We outline how to get the model Hamiltonian in reciprocal space. In real space, the lattice Hamiltonian is defined as

$$
\begin{aligned}
H_{\text {lattice }}= & \frac{M}{2} \sum_{m}\left(\dot{X}_{A, m}^{2}+\dot{X}_{B, m}^{2}\right)+\sum_{m} \frac{K}{2}\left[\left(X_{A, m}-X_{B, m}\right)^{2}\right. \\
& \left.+\left(X_{B, m}-X_{A, m+1}\right)^{2}\right] .
\end{aligned}
$$

Here, the unit cell number is $N$, each unit cell includes two atoms denoted as $\eta=A, B$, and the lattice constant is set as $2 a . X_{\eta, m}$ is the atom coordinate and $m$ is the unit cell index. The first term in Eq. (A1) is the kinetic energy with atom mass $M$, and the second term is the potential energy with the force constant $K$. Denoting $X_{\eta, m}=X_{\eta, m}^{0}+u_{\eta, m}$, where $X_{\eta, m}^{0}$ is the nondistorted position for atom $\eta$ at the $m$ th unit cell and $u_{\eta, m}$ is its displacement from the equilibrium position, then except for a constant term, the lattice Hamiltonian is rewritten as

$$
\begin{aligned}
H_{\text {lattice }}= & \frac{M}{2} \sum_{m}\left(\dot{u}_{A, m}^{2}+\dot{u}_{B, m}^{2}\right) \\
& +\sum_{m} K\left[u_{A, m}^{2}+u_{B, m}^{2}-u_{A, m} u_{B, m}-u_{B, m} u_{A, m+1}\right] .
\end{aligned}
$$

The equation for $u_{\eta, m}$ can be obtained from the Hamiltonian equation as

$$
\begin{aligned}
& \ddot{u}_{A, m}=-\frac{K}{M}\left(2 u_{A, m}-u_{B, m}-u_{B, m-1}\right), \\
& \ddot{u}_{B, m}=-\frac{K}{M}\left(2 u_{B, m}-u_{A, m}-u_{A, m+1}\right) .
\end{aligned}
$$

Due to the translation symmetry, the eigensolution of the above equation with wave vector $q$ is assumed to be

$$
\left(\begin{array}{l}
u_{\mu, q, A}(m, t) \\
u_{\mu, q, B}(m, t)
\end{array}\right)=\frac{1}{\sqrt{M}}\left(\begin{array}{l}
e_{\mu, q, A} \\
e_{\mu, q, B}
\end{array}\right) e^{2 i q m a-i \omega_{\mu, q} t},
$$

where $\mu=\mathrm{a}$,o denotes the two longitudinal phonon branch ("a" for acoustic mode and "o" for optical mode). The equation for $\omega_{\mu, q}$ and $\mathbf{e}_{\mu, q}=\left(e_{\mu, q, A}, e_{\mu, q, B}\right)^{T}$ will be

$$
\left(\begin{array}{cc}
\frac{2 K}{M}-\omega_{\mu, q}^{2} & -\frac{K}{M}\left(1+e^{-2 i q a}\right) \\
-\frac{K}{M}\left(1+e^{2 i q a}\right) & \frac{2 K}{M}-\omega_{\mu, q}^{2}
\end{array}\right)\left(\begin{array}{l}
e_{\mu, q, A} \\
e_{\mu, q, B}
\end{array}\right)=0 .
$$

The dispersion relations for the acoustic and optical phonon branch are respectively

$$
\omega_{\mathrm{a}, q}^{2}=\frac{2 K}{M}(1-\cos q a), \quad \omega_{\mathrm{o}, q}^{2}=\frac{2 K}{M}(1+\cos q a) .
$$

With the notations here, the nesting phonon causing Peierls instability is the optical phonon mode with $q=0$. Its frequency is given as $\Omega^{2}=\frac{4 K}{M}$ and the corresponding vector $\mathbf{e}_{\mathrm{o}, 0}=(1 / \sqrt{2},-1 / \sqrt{2})^{T}$.

Introducing the normal coordinates $Q_{\mu, q}$ and the conjugate momentum $P_{\mu, q}$, where

$$
\begin{aligned}
& u_{\eta, m}(t)=\frac{1}{\sqrt{N M}} \sum_{\mu, q} e_{\mu, q, \eta} Q_{\mu, q}(t) e^{2 i q m a}, \\
& \dot{u}_{\eta, m}(t)=\frac{1}{\sqrt{N M}} \sum_{\mu, q} e_{\mu, q, \eta} P_{\mu, q}(t) e^{2 i q m a},
\end{aligned}
$$


the lattice Hamiltonian becomes

$$
H_{\text {lattice }}=\frac{1}{2} \sum_{\mu, q}\left(P_{\mu, q}^{*} P_{\mu, q}+\omega_{\mu, q}^{2} Q_{\mu, q}^{*} Q_{\mu, q}\right) .
$$

Specially for the nesting phonon, the atom displacement is

$$
u_{A, m}=\frac{\mathcal{Q}}{\sqrt{2 N M}}, \quad u_{B, m}=-\frac{\mathcal{Q}}{\sqrt{2 N M}},
$$

where $\mathcal{Q}$ denotes the normal coordinate for the nesting phonon mode.

From Eq. (A2), we have

$$
\begin{aligned}
Q_{\mu, q}(t) & =\sqrt{\frac{M}{N}} \sum_{\eta, m} e_{\mu, q, \eta} u_{\eta, m}(t) e^{-2 i q m a}, \\
P_{\mu, q}(t) & =\sqrt{\frac{M}{N}} \sum_{\eta, m} e_{\mu, q, \eta} \dot{u}_{\eta, m}(t) e^{-2 i q m a} .
\end{aligned}
$$

The commutation relation to quantize the Hamiltonian (A4) is

$$
\left[\widehat{Q}_{\mu, q}, \widehat{P}_{\mu^{\prime}, q^{\prime}}\right]=i \hbar \delta_{q,-q^{\prime}} \delta_{\mu, \mu^{\prime}},
$$

which can be deduced from the commutation relation $\left[u_{\eta, m}, M \dot{u}_{\eta, m}\right]=i \hbar$.

\section{Electron Hamiltonian}

Now we consider the electron Hamiltonian. In real space, it reads

$$
\begin{aligned}
H= & -\sum_{m}\left[t_{A, m ; B, m}\left(c_{A, m}^{\dagger} c_{B, m}+c_{B, m}^{\dagger} c_{A, m}\right)\right. \\
& \left.+t_{B, m ; A, m+1}\left(c_{B, m}^{\dagger} c_{A, m+1}+c_{A, m+1}^{\dagger} c_{B, m}\right)\right] .
\end{aligned}
$$

The hopping parameter here depends on the atom positions, i.e.,

$$
\begin{gathered}
t_{A, m ; B, m}=t_{0}+\alpha\left(u_{A, m}-u_{B, m}\right), \\
t_{B, m ; A, m+1}=t_{0}+\alpha\left(u_{B, m}-u_{A, m+1}\right),
\end{gathered}
$$

where $t_{0}$ is the hopping parameter when the atoms are at the position $\left\{X_{\eta, m}^{0}\right\}$ without distortion. The spin index has been neglected for convenience.

We first consider the undistorted part

$$
\begin{aligned}
H_{e}= & -t_{0} \sum_{m}\left[c_{A, m}^{\dagger} c_{B, m}+c_{B, m}^{\dagger} c_{A, m}\right. \\
& \left.+c_{B, m}^{\dagger} c_{A, m+1}+c_{A, m+1}^{\dagger} c_{B, m}\right] .
\end{aligned}
$$

Defining the operators for Bloch states as

$$
c_{\eta, k}=\frac{1}{\sqrt{N}} \sum_{m} e^{-2 i k m a} c_{\eta, m}, \quad c_{\eta, k}^{\dagger}=\frac{1}{\sqrt{N}} \sum_{m} e^{2 i k m a} c_{\eta, m}^{\dagger},
$$

then we have

$$
c_{\eta, m}=\frac{1}{\sqrt{N}} \sum_{k} e^{2 i k m a} c_{\eta, k}, \quad c_{\eta, m}^{\dagger}=\frac{1}{\sqrt{N}} \sum_{k} e^{-2 i k m a} c_{\eta, k},
$$

and the Hamiltonian (A7) becomes

$$
H_{e}=-t_{0} \sum_{k}\left[\left(1+e^{-2 i k a}\right) c_{A, k}^{\dagger} c_{B, k}+\left(1+e^{2 i k a}\right) c_{B, k}^{\dagger} c_{A, k}\right] .
$$

\section{Electron-phonon interaction}

Now we consider the interaction between the electron and lattice, where

$$
\begin{aligned}
H_{\mathrm{ep}}= & -\alpha \sum_{m}\left[\left(u_{A, m}-u_{B, m}\right)\left(c_{A, m}^{\dagger} c_{B, m}+c_{B, m}^{\dagger} c_{A, m}\right)\right. \\
& \left.+\left(u_{B, m}-u_{A, m+1}\right)\left(c_{B, m}^{\dagger} c_{A, m+1}+c_{A, m+1}^{\dagger} c_{B, m}\right)\right] .
\end{aligned}
$$

Substituting Eqs. (A2) and (A8) into Eq. (A9), we get

$$
\begin{aligned}
H_{\mathrm{ep}}= & \sum_{\mu, q, k}\left(g_{\mu, q, k} \widehat{Q}_{\mu, q} c_{A, k}^{\dagger} c_{B, k-q}\right. \\
& \left.+g_{\mu,-q,-k} \widehat{Q}_{\mu,-q} c_{B, k-q}^{\dagger} c_{A, k}\right) .
\end{aligned}
$$

Here, the interaction coefficient is given as

$g_{\mu, q, k}=-\frac{\alpha}{\sqrt{N M}}\left[e_{\mu, q, A}\left(1-e^{2 i(q-k) a}\right)-e_{\mu, q, B}\left(1-e^{-2 i k a}\right)\right]$,

and $g_{\mu,-q,-k}=g_{\mu, q, k}^{*}$.

For the nesting phonon mode $(\mathrm{o}, 0)$, we have

$$
g_{\mathrm{o}, 0, k}=-\sqrt{\frac{2}{N M}} \alpha\left(1-e^{-2 i k a}\right),
$$

and the interaction term between the electrons and the nesting phonon will be

$V_{\mathrm{o} e}=-\sqrt{\frac{2 \alpha^{2}}{N M}} \widehat{\mathrm{Q}}\left[\left(1-e^{-2 i k a}\right) c_{A, k}^{\dagger} c_{B, k}+\left(1-e^{2 i k a}\right) c_{B, k}^{\dagger} c_{A, k}\right]$.

We denote $\widehat{\mathrm{Q}} \equiv \widehat{Q}_{\mathrm{o}, 0}$ and $\widehat{\mathrm{P}} \equiv \widehat{P}_{\mathrm{o}, 0}$ for simplicity.

\section{APPENDIX B: DENSITY MATRIX FORMULA}

Here we outline how to deal with the open quantum dynamics with density matrix technique [15]. The whole system is divided into the system part and the bath part, and the interaction between the system and the bath is denoted as $V$. The Hamiltonian is written as

$$
H=H_{S}+H_{B}+V .
$$

The equation for the density matrix of the whole system in the interaction picture (denoted by $\approx$ ) is

$$
\frac{d \widetilde{\rho}}{d t}=\frac{1}{i \hbar}[\tilde{V}, \widetilde{\rho}],
$$

which has the formal solution

$$
\widetilde{\rho}(t)=\widetilde{\rho}\left(t^{\prime}\right)+\frac{1}{i \hbar} \int_{t^{\prime}}^{t} d \tau[\tilde{V}(\tau), \widetilde{\rho}(\tau)] .
$$

Then Eq. (B2) becomes

$$
\begin{aligned}
\frac{d \widetilde{\rho}(t)}{d t}= & \frac{1}{i \hbar}\left[\tilde{V}(t), \widetilde{\rho}\left(t^{\prime}\right)\right]+\left(\frac{1}{i \hbar}\right)^{2} \\
& \times \int_{t^{\prime}}^{t} d \tau[\widetilde{V}(t),[\tilde{V}(\tau), \widetilde{\rho}(\tau)]] .
\end{aligned}
$$

The reduced density matrix for the system is

$$
\tilde{\rho}_{S}(t)=\operatorname{Tr}_{B}[\tilde{\rho}(t)] .
$$


Assuming that

$$
\widetilde{\rho}\left(t^{\prime}\right)=\widetilde{\rho}_{S}\left(t^{\prime}\right) \otimes \widetilde{\rho}_{B}\left(t^{\prime}\right), \quad \widetilde{\rho}(t)=\widetilde{\rho}_{S}(t) \otimes \widetilde{\rho}_{B}\left(t^{\prime}\right)+\widetilde{\rho}_{c}(t), \quad \widetilde{V}=\widetilde{V}_{S} \widetilde{V}_{B},
$$

then keeping to the second order of the interaction, the equation for the reduced density matrix $\tilde{\rho}_{S}(t)$ will be [15]

$$
\begin{aligned}
\frac{d \widetilde{\rho}_{S}(t)}{d t}= & \frac{1}{i \hbar}\left[\widetilde{V}_{S}(t), \widetilde{\rho}_{S}\left(t^{\prime}\right)\right] \operatorname{Tr}_{B}\left[\widetilde{V}_{B} \widetilde{\rho}_{B}\right]+\left(\frac{1}{i \hbar}\right)^{2} \int_{t^{\prime}}^{t} d \tau\left[\widetilde{V}_{S}(t), \widetilde{V}_{S}(\tau) \widetilde{\rho}_{S}(\tau)\right] \operatorname{Tr}_{B}\left[\widetilde{V}_{B}(t) \widetilde{V}_{B}(\tau) \widetilde{\rho}_{B}\left(t^{\prime}\right)\right] \\
& -\left(\frac{1}{i \hbar}\right)^{2} \int_{t^{\prime}}^{t} d \tau\left[\widetilde{V}_{S}(t), \widetilde{\rho}_{S}(\tau) \widetilde{V}_{S}(\tau)\right] \operatorname{Tr}_{B}\left[\widetilde{V}_{B}(\tau) \widetilde{V}_{B}(t) \widetilde{\rho}_{B}\left(t^{\prime}\right)\right] \\
= & \frac{1}{i \hbar}\left\langle\widetilde{V}_{B}\right\rangle\left[\widetilde{V}_{S}(t), \widetilde{\rho}_{S}\left(t^{\prime}\right)\right]+\frac{1}{(i \hbar)^{2}} \int_{t^{\prime}}^{t} d \tau \operatorname{Re}\left\langle\widetilde{V}_{B}(t) \widetilde{V}_{B}(\tau)\right\rangle\left[\widetilde{V}_{S}(t),\left[\widetilde{V}_{S}(\tau), \widetilde{\rho}_{S}(\tau)\right]\right] \\
& +\frac{i}{(i \hbar)^{2}} \int_{t^{\prime}}^{t} d \tau \operatorname{Im}\left\langle\widetilde{V}_{B}(t) \widetilde{V}_{B}(\tau)\right\rangle\left[\widetilde{V}_{S}(t),\left\{\widetilde{V}_{S}(\tau), \widetilde{\rho}_{S}(\tau)\right\}\right] .
\end{aligned}
$$

\section{APPENDIX C: DYNAMICAL EQUATIONS FOR $\mathcal{Q}$ AND $\mathcal{P}$}

We consider the dynamics of the nesting phonon mode $(0,0)$ in the small time interval $\left[t^{\prime}, t\right]$. For the interaction term $V=-\widehat{\mathrm{q}} \otimes \widehat{\mathrm{F}}$ in the body text, we substitute $\widetilde{V}_{S}=\widetilde{\mathrm{q}}$ and $\widetilde{V}_{B}=-\widetilde{\mathrm{F}}$ into Eq. (B6), which gives

$$
\begin{aligned}
\frac{d \widetilde{\rho}_{S}(t)}{d t}= & -\frac{1}{i \hbar}\langle\widetilde{\mathrm{F}}\rangle\left[\widetilde{\mathrm{q}}(t), \widetilde{\rho}_{S}\left(t^{\prime}\right)\right]+\frac{1}{(i \hbar)^{2}} \int_{t^{\prime}}^{t} d \tau \operatorname{Re}\langle\widetilde{\mathrm{F}}(t) \widetilde{\mathrm{F}}(\tau)\rangle\left[\widetilde{\mathrm{q}}(t),\left[\widetilde{\mathrm{q}}(\tau), \widetilde{\rho}_{S}(\tau)\right]\right] \\
& +\frac{i}{(i \hbar)^{2}} \int_{t^{\prime}}^{t} d \tau \operatorname{Im}\langle\widetilde{\mathrm{F}}(t) \widetilde{\mathrm{F}}(\tau)\rangle\left[\widetilde{\mathbf{q}}(t),\left\{\widetilde{\mathrm{q}}(\tau), \widetilde{\rho}_{S}(\tau)\right\}\right] .
\end{aligned}
$$

Then the equation for $\mathcal{Q}(t)=\mathcal{Q}_{0}+\mathrm{q}(t)$ is

$$
\begin{aligned}
& \frac{d \mathcal{Q}(t)}{d t}=\frac{d \mathrm{q}(t)}{d t}=\frac{d}{d t} \operatorname{Tr}[\widetilde{\mathrm{q}}(t) \widetilde{\rho}(t)] \\
& =\operatorname{Tr}\left[\frac{d \widetilde{\mathrm{q}}(t)}{d t} \widetilde{\rho}(t)\right]+\operatorname{Tr}\left[\widetilde{\mathrm{q}}(t) \frac{d \widetilde{\rho}(t)}{d t}\right] \\
& =\operatorname{Tr}\left[\frac{1}{i \hbar}\left[\widetilde{\mathbf{q}}, \widetilde{H}_{S}\right] \widetilde{\rho}(t)\right]-\frac{1}{i \hbar}\langle\widetilde{\mathbf{F}}\rangle \operatorname{Tr}\left[\widetilde{\mathbf{q}}(t)\left[\widetilde{\mathbf{q}}(t), \widetilde{\rho}\left(t^{\prime}\right)\right]\right]+\frac{i}{(i \hbar)^{2}} \int_{t^{\prime}}^{t} d \tau \operatorname{Im}\langle\widetilde{\mathbf{F}}(t) \widetilde{\mathbf{F}}(\tau)\rangle \operatorname{Tr}[\widetilde{\mathbf{q}}(t)[\widetilde{\mathbf{q}}(t),\{\widetilde{\mathbf{q}}(\tau), \widetilde{\rho}(\tau)\}]] \\
& +\frac{1}{(i \hbar)^{2}} \int_{t^{\prime}}^{t} d \tau \operatorname{Re}\langle\widetilde{\mathbf{F}}(t) \widetilde{\mathbf{F}}(\tau)\rangle \operatorname{Tr}[\widetilde{\mathbf{q}}(t)[\widetilde{\mathbf{q}}(t),[\widetilde{\mathbf{q}}(\tau), \widetilde{\rho}(\tau)]]] \\
& =\operatorname{Tr}[\widetilde{P}(t) \widetilde{\rho}(t)] \\
& =\mathcal{P}(t) \text {. }
\end{aligned}
$$

Similarly, the equation for $\mathcal{P}(t)$ is

$$
\begin{aligned}
\frac{d \mathcal{P}(t)}{d t}= & \frac{d}{d t} \operatorname{Tr}[\widetilde{\mathrm{P}}(t) \widetilde{\rho}(t)] \\
= & \operatorname{Tr}\left[\frac{d \widetilde{\mathrm{P}}(t)}{d t} \widetilde{\rho}(t)\right]+\operatorname{Tr}\left[\widetilde{\mathrm{P}}(t) \frac{d \widetilde{\rho}(t)}{d t}\right] \\
= & \operatorname{Tr}\left[\frac{1}{i \hbar}\left[\widetilde{\mathrm{P}}, \widetilde{H}_{S}\right] \widetilde{\rho}(t)\right]-\frac{1}{i \hbar}\langle\widetilde{\mathrm{F}}\rangle \operatorname{Tr}\left[\widetilde{\mathrm{P}}(t)\left[\widetilde{\mathrm{q}}(t), \widetilde{\rho}\left(t^{\prime}\right)\right]\right]+\frac{i}{(i \hbar)^{2}} \int_{t^{\prime}}^{t} d \tau \operatorname{Im}\langle\widetilde{\mathrm{F}}(t) \widetilde{\mathrm{F}}(\tau)\rangle \operatorname{Tr}[\widetilde{\mathrm{P}}(t)[\widetilde{\mathrm{q}}(t),\{\widetilde{\mathrm{q}}(\tau), \widetilde{\rho}(\tau)\}]] \\
& +\frac{1}{(i \hbar)^{2}} \int_{t^{\prime}}^{t} d \tau \operatorname{Re}\langle\widetilde{\mathrm{F}}(t) \widetilde{\mathrm{F}}(\tau)\rangle \operatorname{Tr}[\widetilde{\mathrm{P}}(t)[\widetilde{\mathbf{q}}(t),[\widetilde{\mathbf{q}}(\tau), \widetilde{\rho}(\tau)]]] \\
= & -\Omega^{2} \operatorname{Tr}[\widetilde{\mathrm{q}}(t) \widetilde{\rho}(t)]-\Omega^{2} \mathcal{Q}_{0} \operatorname{Tr}[\widetilde{\rho}(t)]+\langle\widetilde{\mathrm{F}}\rangle \operatorname{Tr}\left[\widetilde{\rho}\left(t^{\prime}\right)\right]-\frac{2}{\hbar} \int_{t^{\prime}}^{t} d \tau \operatorname{Im}\langle\widetilde{\mathrm{F}}(t) \widetilde{\mathrm{F}}(\tau)\rangle \operatorname{Tr}[\widetilde{\mathbf{q}}(\tau) \widetilde{\rho}(\tau)] ;
\end{aligned}
$$

that is,

$$
\frac{d \mathcal{P}(t)}{d t}=-\Omega^{2} \mathcal{Q}(t)+\mathcal{F}-\frac{2}{\hbar} \int_{t^{\prime}}^{t} d \tau \mathcal{K}(t-\tau) \mathrm{q}(\tau)
$$


Here, $\mathcal{F}=\langle\widetilde{\mathrm{F}}\rangle$, and the kernel function $\mathcal{K}(t-\tau)$ is

$$
\mathcal{K}(t-\tau)=\operatorname{Im}\langle\widetilde{\mathrm{F}}(t) \widetilde{\mathrm{F}}(\tau)\rangle=\int_{-\infty}^{+\infty} d \omega \mathcal{K}(\omega) \sin (\omega(t-\tau)) .
$$

Then by the partial integration

$$
\begin{aligned}
\frac{2}{\hbar} \int_{t^{\prime}}^{t} d \tau \mathcal{K}(t-\tau) \mathrm{q}(\tau) & =\int_{-\infty}^{+\infty} d \omega \frac{2}{\hbar} \mathcal{K}(\omega) \int_{t^{\prime}}^{t} d \tau \sin (\omega(t-\tau)) \mathbf{q}(\tau) \\
& =\int_{-\infty}^{+\infty} d \omega \frac{2}{\hbar} \frac{\mathcal{K}(\omega)}{\omega} \int_{t^{\prime}}^{t}[d \cos (\omega(t-\tau))] \mathbf{q}(\tau) \\
& =\int_{-\infty}^{+\infty} d \omega \frac{2}{\hbar} \frac{\mathcal{K}(\omega)}{\omega}\left[\mathbf{q}(t)-\cos \left(\omega\left(t-t^{\prime}\right)\right) \mathbf{q}\left(t^{\prime}\right)\right]-\int_{-\infty}^{+\infty} d \omega \frac{2}{\hbar} \frac{\mathcal{K}(\omega)}{\omega} \int_{t^{\prime}}^{t} \cos (\omega(t-\tau)) \frac{d \mathbf{q}(\tau)}{d \tau} \\
& =\gamma\left(t-t^{\prime}\right) \mathbf{q}\left(t^{\prime}\right)-\gamma(0) \mathbf{q}(t)+\int_{t^{\prime}}^{t} d \tau \gamma(t-\tau) \dot{\mathbf{q}}(\tau),
\end{aligned}
$$

where we denote

$$
\gamma(\omega)=-\frac{2}{\hbar} \frac{\mathcal{K}(\omega)}{\omega}, \quad \gamma(t-\tau)=\int_{-\infty}^{+\infty} d \omega \gamma(\omega) \cos (\omega(t-\tau)) .
$$

According to the assumption, i.e., in the infinitesimal time interval $t \rightarrow t^{\prime}, \mathrm{q}\left(t^{\prime}\right)=0$, and $\mathrm{q}(t) \rightarrow 0$, the equation for $\mathcal{Q}$ finally becomes

$$
\frac{d^{2} \mathcal{Q}}{d t^{2}}=-\int_{t^{\prime}}^{t} d \tau \gamma(t-\tau) \dot{\mathcal{Q}}(\tau)-\Omega^{2} \mathcal{Q}+\mathcal{F}
$$

\section{APPENDIX D: CORRELATION FUNCTION}

Here, we discuss some general properties of the correlation function

$$
\langle\widehat{A}(t) \widehat{A}(\tau)\rangle=\operatorname{Tr}\left[\widehat{A}(t) \widehat{A}(\tau) \rho\left(t^{\prime}\right)\right]
$$

For the partition function $e^{-\beta \Omega}=\operatorname{Tr}\left[e^{-\beta(\widehat{H}-\mu \widehat{N})}\right]$, the density matrix $\rho\left(t^{\prime}\right)$ is $\rho\left(t^{\prime}\right)=e^{\beta \Omega-\beta(\widehat{H}-\mu \widehat{N})}$. Then the correlation function is

$$
\langle\widehat{A}(t) \widehat{A}(\tau)\rangle=e^{\beta \Omega} \operatorname{Tr}\left[e^{i \widehat{H}(t-\tau) / \hbar} \widehat{A} e^{-i \widehat{H}(t-\tau) / \hbar} \widehat{A} e^{-\beta(\widehat{H}-\mu \widehat{N})}\right] .
$$

In the Lehmann spectral representation,

$$
\begin{aligned}
& \langle\widehat{A}(t) \widehat{A}(\tau)\rangle=\sum_{l, m}|\langle l|\widehat{A}| m\rangle|^{2} e^{-\beta\left(E_{l}-\mu N_{l}-\Omega\right)} e^{i\left(E_{l}-E_{m}\right)(t-\tau) / \hbar}, \\
& \langle\widehat{A}(\tau) \widehat{A}(t)\rangle=\sum_{l, m}|\langle l|\widehat{A}| m\rangle|^{2} e^{-\beta\left(E_{m}-\mu N_{m}-\Omega\right)} e^{i\left(E_{l}-E_{m}\right)(t-\tau) / \hbar} .
\end{aligned}
$$

Defining the spectral function

$$
\begin{aligned}
\mathcal{J}(\omega)= & \frac{e^{\beta \Omega}}{2} \sum_{l, m}|\langle l|\widehat{A}| m\rangle|^{2}\left(e^{-\beta\left(E_{l}-\mu N_{l}\right)}+e^{-\beta\left(E_{m}-\mu N_{m}\right)}\right) \\
& \times \delta\left(\frac{E_{l}-E_{m}}{\hbar}-\omega\right),
\end{aligned}
$$

the real part of the correlation function is expressed as

$$
\operatorname{Re}\langle\widehat{A}(t) \widehat{A}(\tau)\rangle=\int_{-\infty}^{+\infty} d \omega \mathcal{J}(\omega) e^{i \omega(t-\tau)} .
$$

Similarly, defining

$$
\begin{aligned}
\mathcal{K}(\omega)= & \frac{e^{\beta \Omega}}{2} \sum_{l, m}|\langle l|\widehat{A}| m\rangle|^{2}\left(e^{-\beta\left(E_{l}-\mu N_{l}\right)}-e^{-\beta\left(E_{m}-\mu N_{m}\right)}\right) \\
& \times \delta\left(\frac{E_{l}-E_{m}}{\hbar}-\omega\right)
\end{aligned}
$$

the imaginary part of the correlation function is

$$
i \operatorname{Im}\langle\widehat{A}(t) \widehat{A}(\tau)\rangle=\int_{-\infty}^{+\infty} d \omega \mathcal{K}(\omega) e^{i \omega(t-\tau)}
$$

For chemical potential $\mu=0$, notice that

$$
\begin{aligned}
\mathcal{K}(\omega)= & \frac{e^{\beta \Omega}}{2} \sum_{l, m}|\langle l|\widehat{A}| m\rangle|^{2} \frac{e^{-\beta E_{l}}-e^{-\beta E_{m}}}{e^{-\beta E_{l}}+e^{-\beta E_{m}}}\left(e^{-\beta E_{l}}+e^{-\beta E_{m}}\right) \\
& \times \delta\left(\frac{E_{l}-E_{m}}{\hbar}-\omega\right) \\
= & \frac{e^{\beta \Omega}}{2} \sum_{l, m}|\langle l|\widehat{A}| m\rangle|^{2} \frac{e^{-\beta\left(E_{l}-E_{m}\right) / 2}-e^{\beta\left(E_{l}-E_{m}\right) / 2}}{e^{-\beta\left(E_{l}-E_{m}\right) / 2}+e^{\beta\left(E_{l}-E_{m}\right) / 2}} \\
& \times\left(e^{-\beta E_{l}}+e^{-\beta E_{m}}\right) \delta\left(\frac{E_{l}-E_{m}}{\hbar}-\omega\right) \\
= & -\mathcal{J}(\omega) \tanh (\beta \hbar \omega / 2) .
\end{aligned}
$$

This relation reflects the fluctuation-dissipation theorem. In the high-temperature limit, $\beta \rightarrow 0$, then $\mathcal{J}(\omega)=-\frac{2}{\beta \hbar \omega} \mathcal{K}(\omega)$. 


\section{APPENDIX E: BATH PROPERTIES}

Here, we calculate the force and the correlation functions for the electron bath. According to (A5), we denote

$$
\Delta=u_{A, m}=-u_{B, m}=\frac{\mathcal{Q}}{\sqrt{2 N M}} .
$$

The Hamiltonian for the electron bath will be

$$
\begin{aligned}
H_{B} & =H_{e}-\mathcal{Q} \widehat{\mathrm{F}} \\
& =\sum_{k, \sigma}\left(c_{A, k, \sigma}^{\dagger}, c_{B, k, \sigma}^{\dagger}\right)\left(\begin{array}{cc}
0 & \Lambda_{k} e^{-i \phi_{k}} \\
\Lambda_{k} e^{i \phi_{k}} & 0
\end{array}\right)\left(\begin{array}{l}
c_{A, k, \sigma} \\
c_{B, k, \sigma}
\end{array}\right),
\end{aligned}
$$

where $\Lambda_{k} e^{-i \phi_{k}} \equiv-t_{0}\left(1+e^{-2 i k a}\right)-2 \alpha \Delta\left(1-e^{-2 i k a}\right)$. Therefore,

$$
\begin{aligned}
\Lambda_{k} & =\sqrt{4 t_{0}^{2} \cos ^{2} k a+16 \alpha^{2} \Delta^{2} \sin ^{2} k a}, \\
\cos \phi_{k} & =-\frac{2 t_{0} \cos ^{2} k a+4 \alpha \Delta \sin ^{2} k a}{\sqrt{4 t_{0}^{2} \cos ^{2} k a+16 \alpha^{2} \Delta^{2} \sin ^{2} k a}}, \\
\sin \phi_{k} & =-\frac{2 t_{0} \sin k a \cos k a-4 \alpha \Delta \sin k a \cos k a}{\sqrt{4 t_{0}^{2} \cos ^{2} k a+16 \alpha^{2} \Delta^{2} \sin ^{2} k a}} .
\end{aligned}
$$

The eigenvalues of $H_{B}$ will be $\epsilon_{ \pm, k, \sigma}= \pm \Lambda_{k}$, with the corresponding eigenvectors

$$
\left(\begin{array}{l}
\lambda_{+, k, \sigma} \\
\lambda_{-, k, \sigma}
\end{array}\right)=\left(\begin{array}{cc}
\frac{1}{\sqrt{2}} & \frac{e^{-i \phi_{k}}}{\sqrt{2}} \\
-\frac{e^{i \phi_{k}}}{\sqrt{2}} & \frac{1}{\sqrt{2}}
\end{array}\right)\left(\begin{array}{l}
c_{A, k, \sigma} \\
c_{B, k, \sigma}
\end{array}\right) .
$$

In terms of $\lambda_{ \pm, k, \sigma}$, the force operator $\widehat{F}$ will be

$$
\begin{array}{r}
\widehat{\mathrm{F}}=- \\
\sum_{k, \sigma}\left[g_{k}\left(\lambda_{+, k, \sigma}^{\dagger} \lambda_{+, k, \sigma}-\lambda_{-, k, \sigma}^{\dagger} \lambda_{-, k, \sigma}\right)\right. \\
\left.+h_{k} \lambda_{+, k, \sigma}^{\dagger} \lambda_{-, k, \sigma}+h_{k}^{*} \lambda_{-, k, \sigma}^{\dagger} \lambda_{+, k, \sigma}\right],
\end{array}
$$

with the coefficients

$$
\begin{aligned}
& g_{k}=-\sqrt{\frac{2 \alpha^{2}}{N M}\left(\cos \phi_{k}-\cos \left(\phi_{k}-2 k a\right)\right),} \\
& h_{k}=-\sqrt{\frac{\alpha^{2}}{2 N M}}\left(1-e^{-2 i k a}\right)\left(1+e^{2 i k a-2 i \phi_{k}}\right) .
\end{aligned}
$$

With the expressions for $\cos \phi_{k}$ and $\sin \phi_{k}$, we further have

$$
\begin{aligned}
g_{k} & =\sqrt{\frac{8 \alpha^{2}}{N M}} \frac{2 \alpha \Delta \sin ^{2} k a}{\sqrt{t_{0}^{2} \cos ^{2} k a+4 \alpha^{2} \Delta^{2} \sin ^{2} k a}}, \\
\cos \left(2 k a-2 \phi_{k}\right) & =\frac{t_{0}^{2} \cos ^{2} k a-4 \alpha^{2} \Delta^{2} \sin ^{2} k a}{t_{0}^{2} \cos ^{2} k a+4 \alpha^{2} \Delta^{2} \sin ^{2} k a}, \\
\sin \left(2 k a-2 \phi_{k}\right) & =\frac{2 t_{0} \alpha \Delta \sin 2 k a}{t_{0}^{2} \cos ^{2} k a+4 \alpha^{2} \Delta^{2} \sin ^{2} k a}, \\
\left|h_{k}\right|^{2} & =\frac{8 \alpha^{2}}{N M} \frac{t_{0}^{2} \sin ^{2} k a \cos ^{2} k a}{t_{0}^{2} \cos ^{2} k a+4 \alpha^{2} \Delta^{2} \sin ^{2} k a} .
\end{aligned}
$$

Now we calculate the force $\langle\widehat{F}\rangle$ and the correlation function $\langle\widehat{\mathrm{F}}(t) \widehat{\mathrm{F}}(\tau)\rangle$. The eigenstates of the electron system are denoted as $|l\rangle=\prod_{\chi, k, \sigma} \otimes\left|n_{\chi, k, \sigma}^{l}\right\rangle$, where $\chi= \pm$, and $n_{\chi, k, s}=0$ or 1 . Then the total energy $E_{l}=\sum_{\chi, k, \sigma} n_{\chi, k, \sigma}^{l} \epsilon_{\chi, k, \sigma}$ and the total electron number $N_{l}=\sum_{\chi, k, \sigma} n_{\chi, k, \sigma}$. Thus the electron-bath induced force is

$$
\begin{aligned}
\langle\widehat{\mathrm{F}}\rangle & =e^{\beta \Omega} \sum_{l}\langle l|\widehat{\mathrm{F}}| l\rangle e^{-\beta E_{l}} \\
& =-e^{\beta \Omega} \sum_{l, k, \sigma} g_{k}\left\langle l\left|\lambda_{+, k, \sigma}^{\dagger} \lambda_{+, k, \sigma}-\lambda_{-, k, \sigma}^{\dagger} \lambda_{-, k, \sigma}\right| l\right\rangle e^{-\beta E_{l}},
\end{aligned}
$$

where

$$
e^{-\beta \Omega}=\sum_{l} e^{-\beta E_{l}}=\prod_{\chi, k, \sigma}\left(1+e^{-\beta \epsilon_{\chi, k, \sigma}}\right)
$$

and

$$
\begin{aligned}
& \sum_{l}\left\langle l\left|\lambda_{\chi, k, \sigma}^{\dagger} \lambda_{\chi, k, \sigma}\right| l\right\rangle e^{-\beta E_{l}} \\
& =\sum_{l} n_{\chi, k, \sigma}^{l} e^{-\beta n_{\chi, k, \sigma}^{l} \epsilon_{\chi, k, \sigma}} \prod_{\chi, k, \sigma}^{\prime} e^{-\beta n_{\chi, k, \sigma}^{l} \epsilon_{\chi, k, \sigma}} \\
& =e^{-\beta \epsilon_{\chi, k, \sigma}} \prod_{\chi, k, \sigma}^{\prime}\left(1+e^{-\beta \epsilon_{\chi, k, \sigma}}\right) .
\end{aligned}
$$

Here, $\prod^{\prime}$ denotes all the other states except for $|\chi, k, \sigma\rangle$. This gives

$$
\langle\widehat{\mathrm{F}}\rangle=-\sum_{k, \sigma} g_{k}\left(\frac{1}{e^{\beta \Lambda_{k}}+1}-\frac{1}{e^{-\beta \Lambda_{k}}+1}\right)=\sum_{k, \sigma} g_{k} \tanh \frac{\beta \Lambda_{k}}{2} .
$$

Now we calculate $\mathcal{K}(\omega)$ defined in Eq. (D1) for $\mu=0$. Since

$$
\begin{aligned}
& \sum_{l, m}|\langle l|\widehat{\mathrm{F}}| m\rangle|^{2}\left(e^{-\beta E_{l}}-e^{-\beta E_{m}}\right) \delta\left(\frac{E_{l}-E_{m}}{\hbar}-\omega\right) \\
& \quad=\sum_{k, \sigma}\left|h_{k}\right|^{2}\left(e^{-\beta \epsilon_{+, k, \sigma}}-e^{-\beta \epsilon_{-, k, \sigma}}\right) \sum_{m}^{\prime} e^{-\beta E_{m}^{\prime}}\left(\delta\left(\frac{\epsilon_{+, k, \sigma}-\epsilon_{-, k, \sigma}}{\hbar}-\omega\right)-\delta\left(\frac{\epsilon_{-, k, \sigma}-\epsilon_{+, k, \sigma}}{\hbar}-\omega\right)\right) \\
& \quad=\sum_{k, \sigma}\left|h_{k}\right|^{2}\left(e^{-\beta \Lambda_{k}}-e^{\beta \Lambda_{k}}\right) \prod_{\chi, k, \sigma}^{\prime}\left(1+e^{-\beta \epsilon_{\chi, k, \sigma}}\right)\left(\delta\left(\frac{2 \Lambda_{k}}{\hbar}-\omega\right)-\delta\left(\frac{2 \Lambda_{k}}{\hbar}+\omega\right)\right),
\end{aligned}
$$


then we get

$$
\mathcal{K}(\omega)=\frac{1}{2} \sum_{k, \sigma}\left|h_{k}\right|^{2} \tanh \frac{\beta \Lambda_{k}}{2}\left(\delta\left(\omega+\frac{2 \Lambda_{k}}{\hbar}\right)-\delta\left(\omega-\frac{2 \Lambda_{k}}{\hbar}\right)\right) .
$$

[1] P. M. Chaikin and T. C. Lubensky, Principles of Condensed Matter Physics (Cambridge University Press, Cambridge, 1995).

[2] H. Kronmüller and M. Fähnle, Micromagnetism and the Microstructure of Ferromagnetic Solids (Cambridge University Press, Cambridge, 2003).

[3] K. Kenji, M. Hase, H. Harima, S. I. Nakashima, M. Tani, K. Sakai, H. Negishi, and M. Inoue, Phys. Rev. B 58, R7484 (1998).

[4] J. Demsar, K. Biljaković, and D. Mihailovic, Phys. Rev. Lett. 83, 800 (1999).

[5] A. Tomeljak, H. Schafer, D. Städter, M. Beyer, K. Biljakovic, and J. Demsar, Phys. Rev. Lett. 102, 066404 (2009).

[6] R. Yusupov, T. Mertelj, V. V. Kabanov, S. Brazovskii, P. Kusar, J.-H. Chu, I. R. Fisher, and D. Mihailovic, Nat. Phys. 6, 681 (2010).

[7] L. Perfetti, P. A. Loukakos, M. Lisowski, U. Bovensiepen, H. Berger, S. Biermann, P. S. Cornaglia, A. Georges, and M. Wolf, Phys. Rev. Lett. 97, 067402 (2006).

[8] F. Schmitt, P. S. Kirchmann, U. Bovensiepen, R. G. Moore, L. Rettig, M. Krenz, J.-H. Chu, N. Ru, L. Perfetti, D. H. Lu, M. Wolf, I. R. Fisher, and Z.-X. Shen, Science 321, 1649 (2008).

[9] F. Schmitt, P. S. Kirchmann, U. Bovensiepen, R. G. Moore, J.-H. Chu, D. H. Lu, L. Rettig, M. Wolf, I. R. Fisher, and Z.-X. Shen, New J. Phys. 13, 063022 (2011).

[10] J. C. Petersen, S. Kaiser, N. Dean, A. Simoncig, H. Y. Liu, A. L. Cavalieri, C. Cacho, I. C. E. Turcu, E. Springate,
F. Frassetto, L. Poletto, S. S. Dhesi, H. Berger, and A. Cavalleri, Phys. Rev. Lett. 107, 177402 (2011).

[11] S. Hellmann, T. Rohwer, M. Kalläne, K. Hanff, C. Sohrt, A. Stange, A. Carr, M. M. Murnane, H. C. Kapteyn, L. Kipp, M. Bauer, and K. Rossnagel, Nat. Commun. 3, 1069 (2012).

[12] H. Y. Liu, I. Gierz, J. C. Petersen, S. Kaiser, A. Simoncig, A. L. Cavalieri, C. Cacho, I. C. E. Turcu, E. Springate, F. Frassetto, L. Poletto, S. S. Dhesi, Z.-A. Xu, T. Cuk, R. Merlin, and A. Cavalleri, Phys. Rev. B 88, 045104 (2013).

[13] G. De Filippis, V. Cataudella, E. A. Nowadnick, T. P. Devereaux, A. S. Mishchenko, and N. Nagaosa, Phys. Rev. Lett. 109, 176402 (2012).

[14] W. P. Su, J. R. Schrieffer, and A. J. Heeger, Phys. Rev. Lett. 42, 1698 (1979).

[15] K. Blum, Density Matrix Theory and Applications (SpringerVerlag, Berlin, Heidelberg, 2012).

[16] J. P. Sethna and S. Kivelson, Phys. Rev. B 26, 3513 (1982).

[17] B. Friedman and W. P. Su, Phys. Rev. B 39, 5152 (1989).

[18] W. P. Su, Phys. Rev. Lett. 74, 1167 (1995).

[19] Y. Wang and F. C. Zhang, Phys. Rev. B 89, 094519 (2014).

[20] G. Grüner, Density Waves in Solids (Addison-Wesley, Reading, 1994).

[21] N. Tsuji, M. Eckstein, and P. Werner, Phys. Rev. Lett. 110, 136404 (2013).

[22] M. Sentef, A. F. Kemper, B. Moritz, J. K. Freericks, Z.-X. Shen, and T. P. Devereaux, Phys. Rev. X 3, 041033 (2013). 